Quantum Field Theory Under the Influence

of External Conditions (QFEXT11)

International Journal of Modern Physics: Conference Series

Vol. 14 (2012) 316-325

(C) World Scientific Publishing Company

DOI: $10.1142 / \mathrm{S} 201019451200743 \mathrm{X}$

\title{
YANG-MILLS CONDENSATES IN COSMOLOGY
}

\author{
D.V. GAL'TSOV \\ Department of Theoretical Physics, Moscow State University, \\ 119899, Moscow, Russia \\ galtsov@physics.msu.ru \\ E.A. DAVYDOV \\ Bogoliubov Laboratory of Theoretical Physics, JINR, \\ 141980, Dubna, Moscow region, Russia \\ davydov@theor.jinr.ru
}

Published 28 July 2012

\begin{abstract}
We discuss homogeneous and isotropic cosmological models driven by $S U(2)$ gauge fields in the framework of Einstein gravity. There exists a Yang-Mills field configuration, parametrized by a single scalar function, which consists of parallel electric and magnetic fields and has the stress tensor mimicking an homogeneous and isotropic fluid. The unique $S U(2)$ gauge theory with spontaneous symmetry breaking sharing the same property is the Yang-Mills coupled to the complex doublet Higgs, this exists only in the case of the closed universe. This model contains an intrinsic mechanism for inflation due to the Higgs potential. Our second goal is to show that a successful inflation can be achieved also within the pure Yang-Mills theory adding an appropriate theta-term.
\end{abstract}

Keywords: Gauge theories; cosmology

\section{Introduction}

A remarkable fact is that the $S U(2)$ Yang-Mills (YM) field admits isotropic and homogeneous configurations parameterized by a single scalar function. This is somewhat surprising since the energy-momentum tensor of a single vector field in flat spacetime always contains anisotropic stresses $E_{i} E_{j}, B_{i} B_{j}$ so this feature is clearly impossible in the $U(1)$ case without spatial averaging. In the $S U(2)$ case one has three vector potentials $A_{\mu}^{a}$ producing electric and magnetic component fields $E_{i}^{a}, B_{i}^{a}$ which make the stress-tensor diagonal and isotropic if $E_{i}^{a}=E \delta_{i}^{a}$ and $B_{i}^{a}=B \delta_{i}^{a}$, indeed $E_{i} E_{j}=E^{2} \delta_{i}^{a} \delta_{j}^{a}=E^{2} \delta_{i j}$. The electric and magnetic vectors are parallel, so both field invariants, the scalar $\mathcal{F}=F_{\mu \nu}^{a} F^{a \mu \nu} / 2=3\left(B^{2}-E^{2}\right)$ and the pseudoscalar $\mathcal{G}=\epsilon^{\mu \nu \lambda \tau} F_{\mu \nu}^{a} F_{\lambda \tau}^{a} / 8=3 E B$ are non-zero.

Such an ansatz can be generalized to any of the FRW metrics

$$
d s^{2}=d t^{2}-a(t)^{2}\left[d \chi^{2}+\Sigma_{k}\left(d \theta^{2}+\sin ^{2} \theta d \phi^{2}\right)\right],
$$


closed $\left(k=1, \Sigma_{1}=\sin \chi\right)$, open $\left(k=-1, \Sigma_{-1}=\sinh \chi\right)$ or spatially flat $(k=$ $\left.0, \Sigma_{0}=r\right)$ and in the matrix form reads ${ }^{1}$

$$
\begin{aligned}
F= & F^{a} T^{a}=\dot{f}\left(T_{n} d t \wedge d \chi+T_{\theta} \Sigma_{k} d t \wedge d \theta+T_{\phi} \Sigma_{k} \sin \theta d t \wedge d \phi\right) \\
& +\Sigma_{k}\left(f^{2}-k\right)\left(T_{\phi} d \chi \wedge d \theta-T_{\theta} \sin \theta d \chi \wedge d \phi+T_{n} \Sigma_{k} \sin \theta d \theta \wedge d \phi\right) .
\end{aligned}
$$

Here the rotating $S U(2)$ generators are used:

$$
T_{n}=\tau^{i} n^{i} / 2 i, T_{\theta}=\tau^{i} e_{\theta}^{i} / 2 i, T_{\phi}=\tau^{i} e_{\phi}^{i} / 2 i
$$

where $n^{i}, e_{\theta}^{i}, e_{\phi}^{i}$ are the spherical unit vectors, and $\tau^{a}$ are Pauli matrices. This property remains valid also for larger gauge groups containing an embedded $\mathrm{SU}(2)^{2,3}$ Remarkably, the EYM cosmology is also solvable at the quantum level, the quantum FRW cosmology having been discussed in a number of papers in the 90-ies. ${ }^{5}$ An interesting feature of the EYM quantum cosmology is a possibility of tunneling transitions between de Sitter and hot FRW cosmologies. ${ }^{6}$ Similar models were formulated in the context of supergravities. ${ }^{7}$ In the spirit of the string theory the Born-Infeld generalizations were also considered. ${ }^{8}$ More recently, the YM cosmological models with modified lagrangians were suggested in the context of the dark energy models. ${ }^{9,10,11}$

Now consider the gauge theory with spontaneous symmetry breaking. For the $S U(2)$ group one has two basic possibilities for the coupled Yang-Mills-Higgs (YMH) system: the real triplet Higgs and the complex doublet Higgs. The non-interacting Higgs belonging to any representation of the gauge group can form isotropic and homogeneous configuration. But for an interacting YM-Higgs system it is not so. It turns out that no homogeneous and isotropic configuration of interacting YMtriple Higgs exist which would be compatible with the Friedman-Robertson-Walker metric for any value of $k$. In the complex doublet case such a configuration exists in the closed case $k=1 .{ }^{12}$ This has a simple explanation: The doublet Higgs has $S O(4)$ symmetry which is the isometry group of the three-sphere. This is another interesting coincidence, which selects the YM-doublet Higgs as natural "realistic" field-theoretical model for cosmology. This model can accommodate for inflation, and at the same time naturally introduces a vector field which could play a role of curvaton. $^{13}$

\section{Yang-Mills-Higgs cosmology}

Consider the EYMH action with complex doublet Higgs:

$$
S=\int\left\{-\frac{1}{16 \pi G} R-\frac{1}{4} F_{\mu \nu}^{a} F^{a \mu \nu}+\frac{1}{2}\left(D_{\mu} \Phi\right)^{\dagger} D_{\mu} \Phi-\frac{\lambda}{4}\left(\Phi^{\dagger} \Phi-v^{2}\right)^{2}\right\} \sqrt{-g} d^{4} x,
$$

where $\Phi$ is the complex doublet Higgs, and $D_{\mu} \Phi=\partial_{\mu} \Phi+g A_{\mu}^{a} T_{a} \Phi$. The YM matrix potential $A_{\mu}=A_{\mu}^{a} T_{a}$ generating the field strength (2) for $k=1$ can be chosen as

$$
A_{\mu}^{a} T_{a}=\frac{1-f(t)}{2 g} U \partial_{\mu} U^{-1}, \quad U=\mathrm{e}^{2 \chi T_{n}},
$$


while an ansatz for Higgs is

$$
\Phi=h(t) U \Phi_{0}, \quad \Phi_{0}^{\dagger} \Phi_{0}=1,
$$

where $\Phi_{0}$ is an arbitrary normalized constant spinor. The compatibility is this configuration with the required spatial symmetries follows from the identity

$$
D_{\mu} \Phi=\frac{1+f(t)}{2} \partial_{\mu} \Phi .
$$

The EYMH action contains three different mass parameters: the Planck mass, the mass of the W-boson, and the Higgs mass

$$
M_{\mathrm{Pl}}=\frac{1}{\sqrt{G}}, \quad M_{W}=g v, \quad M_{H}=\sqrt{\lambda} v .
$$

We rescale the Higgs scalar as $h \rightarrow h M_{\mathrm{Pl}}$ and introduce dimensionless parameters

$$
\alpha=\frac{M_{W}}{g M_{\mathrm{Pl}}}=\frac{v}{M_{\mathrm{Pl}}}, \quad \beta=\frac{M_{H}}{M_{W}}=\frac{\sqrt{\lambda}}{g} .
$$

Finally, introducing the reduced Planck length $l=1 /\left(g M_{\mathrm{Pl}}\right)$, we present the metric in dimensionless terms:

$$
d s^{2}=l^{2}\left\{-N^{2} d t^{2}+a^{2}\left[d \chi^{2}+\sin \chi^{2}\left(d \theta^{2}+\sin ^{2} \theta d \varphi^{2}\right)\right]\right\} .
$$

Substituting the ansatze $(6,5)$, integrating over angles and dividing by the volume of the unit the three-sphere, we obtain the one-dimensional reduced action $S=$ $S_{\mathrm{g}}+S_{\mathrm{YM}}+S_{\mathrm{H}}+S_{\text {int }}$, where

$$
\begin{aligned}
& S_{\mathrm{g}}=\int \frac{3}{8 \pi}\left(a N-\frac{\dot{a}^{2} a}{N}\right) d t, \quad S_{\mathrm{YM}}=\frac{3}{2} \int\left(\frac{\dot{f}^{2} a}{N}-\frac{N\left(f^{2}-1\right)^{2}}{a}\right) d t, \\
& S_{\mathrm{H}}=\int\left(\frac{\dot{h}^{2} a^{3}}{2 N}-\frac{\beta^{2}}{4}\left(h^{2}-\alpha^{2}\right)^{2} N a^{3}\right) d t, \quad S_{\mathrm{int}}=-\frac{3}{4} \int h^{2}(f+1)^{2} N a d t,
\end{aligned}
$$

and the total derivative in the gravitational term is omitted.

Variation with respect to $N$ leads to the constraint equation

$$
\left(H^{2}+\frac{1}{a^{2}}\right)=\frac{8 \pi}{3} \varepsilon, \quad H=\frac{\dot{a}}{a},
$$

and we fix the gauge $N=1$ afterwards. For the energy density we obtain:

$$
\varepsilon=\frac{1}{2} \dot{h}^{2}+\frac{3 \dot{f}^{2}}{2 a^{2}}+V_{f}+V_{h}+V_{\mathrm{int}}
$$

where the potentials read:

$$
V_{f}=\frac{3\left(f^{2}-1\right)^{2}}{2 a^{4}}, \quad V_{h}=\frac{\beta^{2}}{4}\left(h^{2}-\alpha^{2}\right)^{2}, \quad V_{\text {int }}=\frac{3 h^{2}(f+1)^{2}}{4 a^{2}} .
$$

The acceleration equation is obtained by variation of the action over $a$ with account for the constraint:

$$
\frac{\ddot{a}}{a}=-\frac{4 \pi}{3}(\varepsilon+3 p)
$$


leading to the following expression for pressure:

$$
p=\frac{1}{2} \dot{h}^{2}+\frac{\dot{f}^{2}}{2 a^{2}}+\frac{1}{3} V_{f}-V_{h}-\frac{1}{3} V_{\text {int }},
$$

and therefore,

$$
\frac{\ddot{a}}{a}=-\frac{8 \pi}{3}\left(\dot{h}^{2}+\frac{3 \dot{f}^{2}}{2 a^{2}}-V_{h}+V_{f}\right) .
$$

Note that $V_{h}$ and $V_{f}$ enter with different signs, while the interaction term $V_{\text {int }}$ does not enter the acceleration equation at all. The field equations for the YM and Higgs scalar functions read

$$
\begin{aligned}
\ddot{h}+3 H \dot{h} & =-\frac{3}{2 a^{2}} h(f+1)^{2}-\beta^{2}\left(h^{2}-\alpha^{2}\right) h, \\
\ddot{f}+H \dot{f} & =-\frac{1}{2} h^{2}(f+1)-\frac{2}{a^{2}}\left(f^{2}-1\right) f .
\end{aligned}
$$

It is worth noting, that two roots $f= \pm 1$ of the YM potential $V_{f}$ correspond to two neighboring topological vacua. To show this, consider the Chern-Simons 3-form

$$
\omega_{3}=\frac{g^{2}}{8 \pi^{2}} \operatorname{Tr}\left(A \wedge d A-\frac{2 i e}{3} A \wedge A \wedge A\right),
$$

satisfying the equation

$$
d \omega_{3}=\frac{e^{2}}{8 \pi^{2}} \operatorname{Tr} F \wedge F .
$$

Substituting here the potential (5) one finds that it is non-trivial in the closed universe $k=1$, giving the winding number of the map $S U(2) \rightarrow S^{3}$ :

$$
N_{C S}=\int_{S^{3}} \omega_{3}=\frac{1}{4}(f+1)^{2}(2-f) .
$$

The vacuum $f=-1$ is therefore topologically trivial: $N_{C S}=0$, while the vacuum $f=1$ is the next non-trivial one with $N_{C S}=1$. This does not mean, however, that both will be classical solutions to our system.

One can see that the above equations have the following particular solutions:

- If Higgs is in the false vacuum $h=0$, we have the EYM system with the cosmological constant $\Lambda=\alpha^{4} \beta^{2} / 4$; the corresponding scale factor can be found in a closed form. ${ }^{12}$ The Euclidean sector of the same theory contains instanton and wormhole solutions interpolating between the de Sitter and the hot universes. ${ }^{4}$

- A particular (unstable) analytic solution is cosmological sphaleron in the false Higgs vacuum:

$$
h=0, \quad f=0, \quad a^{2}=\frac{\sqrt{6}}{\beta \alpha^{2}} .
$$

This solution describes the YM field sitting at the top of the potential barrier separating two topologically neighboring YM vacua (note that $f=0$ is not vacuum for YM ) and generalizes the pure EYM sphaleron of Ref. 14. In this case $H=0$ and the universe is in the steady state. 
- There is another non-trivial steady state with non-zero $f, h$ :

$$
f=\frac{\sqrt{8} \beta-\sqrt{3}}{\sqrt{8} \beta+\sqrt{3}}, \quad h=\sqrt{f} \alpha, \quad a^{2}=\frac{4(1-f)}{\alpha^{2}} .
$$

- If YM field is in the trivial vacuum $f=-1$, we have a pure Higgs $k=1$ cosmology, which is the basis of the standard inflationary models.

At the same time, there are several regimes which could be expected, but actually are not realized as classical solutions of the EYMH system:

- The system never reduces to the pure EYM cosmology with the conformal equation of state $p=\frac{1}{3} \varepsilon$ and the hot universe metric as described in Ref. 1 .

- The non-trivial vacuum $f=1$ is not a solution to our system: the interaction term does not vanish in this case, so the YM equation will not be satisfied. Thus, the YM field dynamically coupled to Higgs will have the unique vacuum state $f=-1$.

- The Higgs vacuum $h=\alpha$ is not a solution unless the YM field is vacuum $f=-1$.

The system possesses the slow-roll regime when kinetic terms $\dot{f}, \dot{h}$ are small relative to potential terms. There are three different slow-roll regimes:

- inflation, with an approximate equation of state $p=-\varepsilon$ in the case of the dominant Higgs potential $V_{h}$;

- hot universe with $p=\varepsilon / 3$ in the case of dominant YM "potential" $V_{f}$;

- zero-acceleration regime with the equation of state $p=-\varepsilon / 3$ in the case of dominant interaction term $V_{\text {int }}$. The emergence of such an equation of state (usually referred as the string gas equation) regime is somewhat unexpected, it also occurs in the high energy limit of the non-Abelian Born-Infeld cosmology. ${ }^{8}$

Note, that $V_{f}$ and $V_{\text {int }}$ are not really the potentials: they depend on the cosmological scale factor.

The solutions (23) and (24) are fixed points of the six-dimensional dynamical system associated with the equations of motion. The first one corresponds to the local maximum of the Higgs potential, in which case interaction between the Higgs and the YM fields is switched off. The second point corresponds to the minimum of the potential $h_{\min }<\alpha$, shifted by interaction with YM. Correspondingly, the position of the YM function $f$ is also shifted from the extrema of $V_{f}$. In this case the interaction plays a crucial role. The analysis of perturbations around the fixed points shows that the first one is an unstable node. For the second fixed point one obtains stable and unstable directions in the phase space depending on the value of the parameter $\beta$, see Ref. 12 for details. The most interesting case corresponds to stability of this point against small perturbation, which translates in the existence of long quasistable states which terminates by an inflationary period. The number of $e$-folds during inflation depends on the initial value of the Higgs field in the quasistationary phase. 
Qualitatively, behavior of the integral curves obtained in numerical experiments $^{12}$ is somewhat reminiscent of the hybrid inflation with two truly scalar fields. The interaction potential $V_{\text {int }}$ plays the role of trapping potential which drives the inflaton to climb the Higgs potential. As a result, the YM field can substantially enhance the scalar inflation.

\section{Theta-inflation}

Consider now the pure YM theory described by the action $S=\int L \sqrt{-g} d^{4} x$, where the lagrangian $L(\mathcal{F}, \mathcal{G})$ depends arbitrarily on two YM invariants

$$
\mathcal{F}=-F_{\mu \nu}^{a} F^{a \mu \nu} / 2, \quad \mathcal{G}=-\tilde{F}^{a \mu \nu} F_{\mu \nu}^{a} / 4, \quad \tilde{F}^{a \mu \nu}=\frac{\epsilon^{\mu \nu \lambda \tau} F_{\lambda \tau}^{a}}{2 \sqrt{-g}} .
$$

We will focus here on the dependence of the lagrangian on the pseudoscalar $\mathcal{G}$. In gauge theories the linear in $\mathcal{G}$ term is induced by instantons and is called theta-term. We will assume, however, more general dependence of $L$ on $\mathcal{G}$, motivated, e.g. by vacuum polarization.

In the Refs. 10, 12 we derived the equation of state for FRW cosmologies driven by the YM field configuration (2) for a general lagrangian $L(\mathcal{F}, \mathcal{G})$. The contribution coming from $\mathcal{G}$ was found to produce the energy density and pressure with the ratio $w=\varepsilon / p=-1$. It is not difficult to show that this does not depend on the particular configuration of the YM field. The linear functional $S_{\mathcal{G}}=\int \mathcal{G} \sqrt{-g} d^{4} x$ does not depend on the metric:

$$
S_{\mathcal{G}}=-\frac{1}{2} \int \frac{\epsilon^{\mu \nu \lambda \tau}}{\sqrt{-g}} F_{\mu \nu} F_{\lambda \tau} \sqrt{-g} d^{4} x=-\frac{1}{2} \int \epsilon^{\mu \nu \lambda \tau} F_{\mu \nu} F_{\lambda \tau} d^{4} x
$$

and hence does not contribute to the energy-momentum tensor. However, for more general dependence of the lagrangian on $\mathcal{G}$, it is not so, since

$$
\frac{\partial \mathcal{G}}{\partial g^{\mu \nu}}=\frac{1}{2} \mathcal{G} g_{\mu \nu}
$$

Using this, we obtain the YM energy-momentum tensor in the form

$$
T_{\mu \nu}=\frac{2}{\sqrt{-g}} \frac{\delta S}{\delta g^{\mu \nu}}=2 \frac{\partial L}{\partial \mathcal{F}} \frac{\partial \mathcal{F}}{\partial g^{\mu \nu}}+\left(\frac{\partial L}{\partial \mathcal{G}} \mathcal{G}-L\right) g_{\mu \nu},
$$

where the second term looks like the variable cosmological constant. The field invariants for the YM configuration (2) read

$$
\mathcal{F}=\frac{3 \dot{f}^{2}}{a^{2} N^{2}}-\frac{3}{a^{4}}\left(k-f^{2}\right)^{2}, \quad \mathcal{G}=\frac{3 \dot{f}\left(k-f^{2}\right)}{N a^{3}} .
$$

We also introduce the effective electric and magnetic fields

$$
\mathcal{E}=\frac{\dot{f}}{a N}, \quad \mathcal{H}=\frac{k-f^{2}}{a^{2}} \quad \text { so that } \quad \mathcal{F}=3\left(\mathcal{E}^{2}-\mathcal{H}^{2}\right), \quad \mathcal{G}=3 \mathcal{E} \mathcal{H}
$$


Then in the context of the FRW models we find for the energy density and pressure:

$$
\begin{aligned}
& \varepsilon=-L+3 \mathcal{E}\left(2 \mathcal{E} \frac{\partial L}{\partial \mathcal{F}}+\mathcal{H} \frac{\partial L}{\partial \mathcal{G}}\right), \\
& p=L+2\left(2 \mathcal{H}^{2}-\mathcal{E}^{2}\right) \frac{\partial L}{\partial \mathcal{F}}-3 \mathcal{E} \mathcal{H} \frac{\partial L}{\partial \mathcal{G}} .
\end{aligned}
$$

Consider now the lagrangian with a "potential" depending on $\mathcal{G}$ :

$$
L(\mathcal{F}, \mathcal{G})=\frac{\mathcal{F}}{2}-V(\mathcal{G})
$$

Then

$$
\begin{gathered}
\varepsilon_{\mathcal{F}}=\frac{3}{2}\left(\mathcal{E}^{2}+\mathcal{H}^{2}\right), \quad \varepsilon_{\mathcal{G}}=V-3 \mathcal{E} \mathcal{H} V^{\prime}, \\
\varepsilon=\left(\varepsilon_{\mathcal{F}}+\varepsilon_{\mathcal{G}}\right), \quad p=\left(\frac{\varepsilon_{\mathcal{F}}}{3}-\varepsilon_{\mathcal{G}}\right),
\end{gathered}
$$

and the Friedman equations read:

$$
\begin{aligned}
H^{2}+\frac{k}{a^{2}} & =\frac{8 \pi}{3}\left(\varepsilon_{\mathcal{F}}+\varepsilon_{\mathcal{G}}\right) \\
\frac{\ddot{a}}{a}=\dot{H}+H^{2} & =\frac{8 \pi}{3}\left(\varepsilon_{\mathcal{G}}-\varepsilon_{\mathcal{F}}\right) .
\end{aligned}
$$

The dynamics of the YM field in this model is governed by the equation:

$$
\frac{d}{d t}\left(\frac{a^{3}}{2} \frac{\partial \mathcal{F}}{\partial \dot{f}}-a^{3} V^{\prime} \frac{\partial \mathcal{G}}{\partial \dot{f}}\right)=a^{3}\left(\frac{1}{2} \frac{\partial \mathcal{F}}{\partial f}-V^{\prime} \frac{\partial \mathcal{G}}{\partial f}\right) .
$$

Using the relation $V^{\prime \prime}=-\frac{\varepsilon_{\mathcal{G}}^{\prime}}{3 \mathcal{E} \mathcal{H}}$, where primes denote derivatives with respect to $\mathcal{G}$, after some rearrangements we obtain:

$$
\left[1+\varepsilon_{\mathcal{G}}^{\prime} \frac{\mathcal{H}}{\mathcal{E}}\right] \ddot{f}+\left[1-3 \varepsilon_{\mathcal{G}}^{\prime} \frac{\mathcal{H}}{\mathcal{E}}\right] H \dot{f}=2 f \mathcal{H}\left[1+\varepsilon_{\mathcal{G}}^{\prime} \frac{\mathcal{E}}{\mathcal{H}}\right] .
$$

We will investigate the regime when $\varepsilon_{\mathcal{G}}^{\prime}$ terms dominates in the squared brackets, then the Eq. (36) is simplified to

$$
\ddot{f}-3 H \dot{f}=2 f \frac{\mathcal{E}^{2}}{\mathcal{H}} .
$$

Contrary to the scalar inflation generated by the potential term, here the desired regime rather corresponds to the kinetic (electric) term being of the same order as the potential term (magnetic). Indeed, the theta-term, whose dominance produces inflation, is the product of the electric and magnetic components. The dynamics will depend on the initial state of the system, characterized by the ratio $\mathcal{H} / \mathcal{E}$, which should not be too small or too large. Let us investigate the slow-roll regime, which is expected if

- Hubble friction term in the YM equation is dominant;

- the accelerating term in the Friedman equations is dominant: $\varepsilon_{\mathcal{F}} \ll \varepsilon_{\mathcal{G}}$;

- the Hubble parameter is varying slowly, $\epsilon \equiv-\dot{H} / H^{2} \ll 1, \eta \equiv-\ddot{H} /(2 H \dot{H}) \ll 1$;

- the curvature term is negligible $k / a^{2} \ll H^{2}$. 
In what follows we set $k=0$, just noting that the case $k=1$ is rather different and very interesting, it will be considered elsewhere. For the slow-roll approximation it may be not correct to use just $\ddot{f} \ll 3 H \dot{f}$. It will be better to work with the ratio $\psi=f / a$, which is connected with the magnetic component via $\mathcal{H}=-\psi^{2}$ and whose derivative is related to the electric one: $\mathcal{E}=\dot{\psi}+H \psi$. Thus we arrive at the system

$$
\begin{gathered}
\ddot{\psi}+3 H \dot{\psi}=-\psi\left(\dot{H}+2 \frac{\dot{\psi}^{2}}{\psi^{2}}\right), \\
H^{2}=\frac{8 \pi}{3}\left(\varepsilon_{\mathcal{F}}+\varepsilon_{\mathcal{G}}\right), \quad \dot{H}+H^{2}=\frac{8 \pi}{3}\left(\varepsilon_{\mathcal{G}}-\varepsilon_{\mathcal{F}}\right) .
\end{gathered}
$$

The dominance of the theta-terms $\varepsilon_{\mathcal{G}} \gg \varepsilon_{\mathcal{F}}$ implies smallness of the first slow-roll parameter:

$$
\epsilon=\frac{2 \varepsilon_{\mathcal{F}}}{\varepsilon_{\mathcal{F}}+\varepsilon_{\mathcal{G}}} \ll 1 .
$$

To simplify further calculations we proceed with the potential

$$
V(\mathcal{G})=-\theta \mathcal{G} \ln \mathcal{G}
$$

in which case the theta-energy density $\varepsilon_{\mathcal{G}}=\theta \mathcal{G}$ is linear in $\mathcal{G}$, the same as the standard $\mathcal{F}$ term. Then the condition $\varepsilon_{\mathcal{F}} \ll \varepsilon_{\mathcal{G}}$ of the accelerated expansion can be satisfied in a wide range of initial conditions and will persist long enough if $\theta \gg 1$. In this case the condition $\varepsilon_{\mathcal{G}}^{\prime}=\theta \gg 1$ is satisfied and we arrive at the system (38) admitting an accelerated solution if the electric and magnetic components are of the same order.

To ensure slow variation of the Hubble parameter $H$ and the effective inflaton $\psi$ during the inflation one needs slowness of the parameter $\delta=-\frac{\dot{\psi}}{H \psi}$, in terms of which the electric and the magnetic components read

$$
\mathcal{E}=(1-\delta) H \psi, \quad-\frac{\dot{\mathcal{E}}}{H \mathcal{E}}=\frac{\dot{\delta}}{H(1-\delta)}+\delta+\epsilon, \quad-\frac{\dot{\mathcal{H}}}{H \mathcal{H}}=2 \delta .
$$

By virtue of the Friedman equations, $\dot{H} \sim \varepsilon_{\mathcal{F}}$, so we can rewrite the system (38) as

$$
\begin{aligned}
& \frac{\dot{\delta}}{H}=-3 \delta-\epsilon+\delta \epsilon+3 \delta^{2}, \quad \epsilon=2\left[1+\frac{2 \theta \mathcal{E} \mathcal{H}}{\mathcal{E}^{2}+\mathcal{H}^{2}}\right]^{-1} \\
& \eta=\frac{1}{\mathcal{E}^{2}+\mathcal{H}^{2}}\left[\mathcal{E}^{2}\left(\frac{\dot{\delta}}{H(1-\delta)}+\delta+\epsilon\right)+2 \mathcal{H}^{2} \delta\right]
\end{aligned}
$$

To ensure slow variation of $\delta, \dot{\delta} /(H \delta) \ll 1$, we set $\delta=-\epsilon / 3+O\left(\epsilon^{2}\right)$. Then the last equation in the leading order in $\epsilon$ reduces to

$$
\eta=\frac{\epsilon}{3} \frac{\mathcal{E}^{2}-\mathcal{H}^{2}}{\mathcal{E}^{2}+\mathcal{H}^{2}}
$$

therefore $\eta \sim \epsilon, \dot{\epsilon} \sim H \epsilon^{2}$. Finally, smallness of $\epsilon$ implies the constraint on the initial state and the parameter $\theta$ :

$$
\frac{\mathcal{E}^{2}+\mathcal{H}^{2}}{\theta \mathcal{E H}} \ll 1
$$

so $\theta$ should be large enough, with both electric and magnetic fileds non-vanishing. 
The number of $e$-folds is given by

$$
N_{e}=\int_{t_{i}}^{t_{e}} H d t=-\int_{H_{i}}^{H_{e}} \frac{d H}{\epsilon H} .
$$

Using the relation between variations of $\psi$ and $H$ due to $\delta=-\epsilon / 3$, we obtain

$$
\left(\frac{\psi}{\psi_{i}}\right)^{3}=\frac{H_{i}}{H}
$$

where the initial values must satisfy the constraint

$$
H_{i}^{2} \simeq \frac{8 \pi}{3} \varepsilon_{\mathcal{G}} \simeq-8 \pi \theta H_{i} \psi_{i}^{3}, \quad \Rightarrow \quad H=-\frac{8 \pi \theta \psi_{i}^{6}}{\psi^{3}}
$$

The slow roll ends when the condition (44) fails. During the inflation the Hubble parameter decreases since $\dot{H}=-16 \pi \varepsilon_{\mathcal{F}} / 3$, therefore the inflaton $|\psi|$ goes up. The electric component decreases as $\mathcal{E} \simeq H \psi \sim 1 / \psi^{2}$, while the magnetic one increases as $|\mathcal{H}| \sim \psi^{2}$. The slow roll regime stops when the ratio $\mathcal{H} / \mathcal{E}$ approaches the value $\theta$, i.e. the imbalance between the electric and the magnetic components becomes comparable with the imbalance of the standard and $\theta$-term in the lagrangian. This happens when

$$
\frac{\mathcal{H}\left(\psi_{e}\right)}{\mathcal{E}\left(\psi_{e}\right)} \sim \theta \quad \Rightarrow \quad \psi_{e}^{4}=8 \pi \theta^{2} \psi_{i}^{6}
$$

Then the dominance of the $\varepsilon_{\mathcal{G}}^{\prime} \mathcal{E} / \mathcal{H}$ term at the right hand side of the Eq. (36) ends, and the system dynamics changes.

To estimate the number of $e$-folds, we substitute $d H / H=-3 d \psi / \psi$ into (45) using the Eq. (48) to relate $\psi_{e}$ and $\psi_{i}$. For $\epsilon$ use the system (42) expressing it in terms of $\psi$ alone via (46). During the main part of the inflation stage one has $\mathcal{H}>\mathcal{E}$, so that $1 / \epsilon \simeq \theta \mathcal{E} / \mathcal{H}=-\theta H_{i} \psi_{i}^{3} / \psi^{4}$. Finally we obtain:

$$
N_{e} \approx-3 \theta H_{i} \psi_{i}^{3} \int_{\psi_{i}}^{\psi_{e}} \frac{d \psi}{\psi^{5}}=6 \pi \theta^{2} \psi_{i}^{6}\left(\frac{1}{\psi_{i}^{4}}-\frac{1}{\psi_{e}^{4}}\right)=6 \pi \theta^{2} \psi_{i}^{2}-\frac{3}{4} .
$$

Unlike the scalar case when the scalar field is rolling down, the YM inflaton $\psi$ is rolling up during the inflation. To avoid going beyond the Planck mass we should properly choose the initial conditions. Taking as the value of $\psi_{e}$ the rescaled Planck mass $g M_{\mathrm{Pl}}$, we obtain from (48) for the initial value in the same units: $\left|\psi_{i}\right|=$ $\left(8 \pi \theta^{2}\right)^{-1 / 6}<\left|\phi_{e}\right| \sim 1$. Hence the number of $e$-folds will be

$$
N_{e} \approx \frac{3}{4}\left(8 \pi \theta^{2}\right)^{2 / 3} \approx 6.4|\theta|^{4 / 3}
$$

Therefore we need the parameter $\theta$ to be just the order of ten to obtain the slow roll regime with sufficient number of $e$-folds. The strongest inflation takes place when electric and magnetic YM components initially are of the same order. 


\section{Outlook}

Incorporating gauge field into cosmological setting seems natural and promising. We have shown that homogeneity and isotropy selects the YM-doublet Higgs as the unique gauge theory with spontaneous symmetry breaking compatible with the closed FRW cosmology which admits both the inflationary and the hot universe regimes. Our second proposal consists in using a suitable theta-term to achieve cosmic acceleration with the pure YM lagrangian.

\section{Acknowledgments}

D.G. thanks the organizers of QFEXT-11 for invitation and support, and A. Starobinsky, V. Mostepanenko, C. Schubert and S. Gavrilov for useful discussions. This work was supported by RFBR grants 11-02-01371-a and 11-02-01335-a.

\section{References}

1. D. V. Galtsov and M. S. Volkov, Phys. Lett. B 256, 17 (1991).

2. P. V. Moniz and J. M. Mourao, Class. Quant. Grav. 8, 1815 (1991).

3. B. K. Darian and H. P. Kunzle, J. Math. Phys. 38, 4696 (1997).

4. E. E. Donets and D. V. Galtsov, Phys. Lett. B 294, 44 (1992).

5. M. Cavaglia and V. de Alfaro, Mod. Phys. Lett. A 9, 569 (1994); O. Bertolami and P. V. Moniz, Nucl. Phys. B 439, 259 (1995) D. Kapetanakis, G. Koutsoumbas, A. Lukas and P. Mayr, Nucl. Phys. B 433, 435 (1995).

6. A. Hosoya and W. Ogura, Phys. Lett. B 225, 117 (1989); Y. Verbin and A. Davidson, Phys. Lett. B 229, 364 (1989); E. E. Donets and D. V. Galtsov, Phys. Lett. B 294, 44 (1992).

7. P. V. Moniz, Helv. Phys. Acta 69, 293 (1996).

8. V. V. Dyadichev, D. V. Gal'tsov, A. G. Zorin and M. Y. Zotov, Phys. Rev. D 65, 084007 (2002); E. Elizalde, J. E. Lidsey, S. Nojiri, and S. D. Odintsov, Physics Letters B, 571, 1 (2003); A. Fuzfa and J. M. Alimi, Phys. Rev. D 73, 023520 (2006); Phys. Rev. Lett. 97, 061301 (2006).

9. W. Zhao and Y. Zhang, Class. Quant. Grav. 23, 3405 (2006); Y. Zhang, T. Y. Xia, \& W. Zhao, Class. Quant. Grav. 24, 3309 (2007) ; K. Bamba, S. Nojiri and S. D. Odintsov, Phys. Rev. D 77, 123532 (2008); W. Zhao, Int. J. Mod. Phys. D 17, 1245 (2008); M. L. Tong, Y. Zhang, \& T. Y. Xia, ibid, D 18, 797 (2009); W. Zhao, ibid, D18, 1331 (2009); W. Zhao, Y. Zhang and M. L. Tong, "Quantum Yang-Mills Condensate Dark Energy Models," arXiv:0909.3874 [astro-ph.CO]; E. Elizalde and A. J. Lopez-Revelles, Phys. Rev. D 82, 063504 (2010). A. Banijamali and B. Fazlpour, Eur. Phys. J. C 71, 1684 (2011); V. K. Shchigolev, Grav. Cosmol. 17, 272 (2011); Z. W. Fu, Y. Zhang and M. L. Tong, Class. Quant. Grav. 28, 225017 (2011).

10. D. V. Gal'tsov, "Non-Abelian condensates as alternative for dark energy," in 2008 Cosmology, XLIII Rencontres de Moriond, Gioi Publ, 2008, pp. 303 , arXiv:0901.0115 [gr-qc].

11. A. Maleknejad and M. M. Sheikh-Jabbari, Phys. Rev. D 84, 043515 (2011).

12. D. V. Gal'tsov and E. A. Davydov, "Cosmological models with gauge fields," in Proc. Steklov Inst. Math. 272, 119 (2011) [arXiv:1012.2861 [gr-qc]].

13. K. Dimopoulos, M. Karciauskas and J. M. Wagstaff, Phys. Rev. D 81, 023522 (2010).

14. G. W. Gibbons and A. R. Steif, Phys. Lett. B 320, 245 (1994). 